Article

\title{
Prognostic Significance of JMJD3 Expression in Pleural Mesotheliomas
}

\author{
Lauren Rask-Nielsen ${ }^{1}$, Sarita Prabhakaran ${ }^{1}\left(\mathbb{D}\right.$, Ashleigh J. Hocking ${ }^{1}$, Matthew Hussey ${ }^{2}$ and Sonja Klebe ${ }^{1,2, *(D)}$ \\ 1 Department of Anatomical Pathology, College of Medicine and Public Health, Flinders University, \\ Adelaide, SA 5042, Australia; rask0002@flinders.edu.au (L.R.-N.); sarita.prabhakaran@flinders.edu.au (S.P.); \\ ash.hocking@flinders.edu.au (A.J.H.) \\ 2 Department of Surgical Pathology, SA Pathology at Flinders Medical Centre, Adelaide, SA 5042, Australia; \\ matthew.hussey@sa.gov.au \\ * Correspondence: sonja.klebe@sa.gov.au; Tel.: +61-08-82043936
}

Citation: Rask-Nielsen, L.

Prabhakaran, S.; Hocking, A.J.;

Hussey, M.; Klebe, S. Prognostic Significance of JMJD3 Expression in Pleural Mesotheliomas. J. Mol. Pathol. 2021, 2, 223-232. https://doi.org/ $10.3390 /$ jmp2030019

Academic Editor: Giancarlo Troncone

Received: 9 May 2021

Accepted: 30 June 2021

Published: 3 July 2021

Publisher's Note: MDPI stays neutral with regard to jurisdictional claims in published maps and institutional affiliations.

Copyright: (c) 2021 by the authors. Licensee MDPI, Basel, Switzerland. This article is an open access article distributed under the terms and conditions of the Creative Commons Attribution (CC BY) license (https:// creativecommons.org/licenses/by/ $4.0 /)$.
Abstract: Pleural mesothelioma is a disease associated with asbestos exposure and patients often have poor prognosis. Biomarkers that can stratify tumours more efficiently are much sought after to enable more personalized treatment options and predict prognosis. Jumonji domain-containing protein D3 (JMJD3) has variable expression in a range of tumours. However, there has been much discordance in the immunohistochemical labelling of JMJD3 between cancers at different sites and ambiguity exists regarding its functional significance. Recent evidence suggests that although nuclear expression of JMJD3 has a demethylase role in most cancers, there are also demethylase-independent actions of JMJD3 that need to be explored including its cytoplasmic expression. We analysed JMJD3 labelling in 99 pleural mesothelioma tissues and correlated nuclear and cytoplasmic expression with survival outcomes. We found that low nuclear and high cytoplasmic expression were associated with poor survival outcomes in our cohort ( $p=0.014$ and $p=0.041$, respectively). Additionally, we found that low nuclear expression of JMJD3 was frequent in the sarcomatoid subtype $(p<0.001)$. Finally, we showed that cytoplasmic labelling is an independent prognostic marker of poor survival. Our cohort only contained a small number of tumours with high cytoplasmic expression of JMJD3, and a larger cohort study may provide clearer stratification.

Keywords: JMJD3; immunohistochemistry; pleural; mesothelioma

\section{Introduction}

Pleural mesothelioma (PM) is a rare and often devastating type of tumour with poor prognosis. The number of new cases worldwide according to Globocan 2020 is 30,870 with the highest incidence in Europe 13,648 (44.2\%) followed by Asia 9735 (31.5\%) and Northern America 4119 (13.3\%). Lower incidence rates are noted in Africa and Oceania 1119 (3.6\%) and 1101 (3.4\%), respectively. Age standardized (world) incidence rates were 2.4 and 0.46 for males and females, respectively, in Europe, followed by 2.2 and 0.57 in Australia and New Zealand, while the lowest reported age-standardised incidence rates were from Africa (0.09 and 0.03) and the Caribbean (0.08 and 0.02) for males and females, respectively [1]. In 2019, 724 deaths due to mesothelioma were recorded in Australia, with most patients being male. Mesothelioma has been strongly linked to both occupational and non-occupational asbestos exposure. Despite aggressive therapies, survival rates are appreciably lower in comparison to other cancer types. Further contributing to poor outcomes, mesothelioma is often diagnosed at an advanced stage of disease [2].

Histological subtype in combination with TNM staging has formed the basis to determining treatment options and prognostic outcomes. The histological subtype of mesothelioma is well established as a strong predictor of patient outcomes with the epithelioid subtype exhibiting more favourable outcomes when compared to biphasic and sarcomatoid 
histologies [3]. Accurate diagnosis of malignant mesothelioma also requires immunohistochemistry (IHC) which has a major role in morphologically challenging cases and in biopsy and cytology specimens, where tumour architecture is difficult to evaluate. The current guidelines recommend a panel of immunohistochemical markers including two mesothelial (e.g., calretinin, CK5/6, WT-1, or D2-40) and two epithelial markers (e.g., MOC-31 and claudin-4) that offer good sensitivity and specificity [4-6]. Panels must be modified for diagnosis, as mesothelioma often presents with an acellular/paucicellular effusion where the role of IHC for diagnosis of sarcomatoid mesothelioma is somewhat more limited [7]. IHC for BAP1, MTAP or FISH for CDKN2A homozygous deletion can be useful for determining malignancy and is especially useful in cytology samples as cytological material is usually the first and only sample available in the diagnosis of mesothelioma, hence the use of immunocytochemical biomarkers with high sensitivity and specificity are desirable [8].

$\mathrm{H} 3 \mathrm{~K} 27 \mathrm{me} 3$ is an epigenetic modification to the DNA packaging protein Histone $\mathrm{H} 3$. The Jumonji domain-containing protein D3 (JMJD3/KDM6B) catalyses the demethylation of H3K27me3 and H3K27me2 to remove methyl groups from lysine 27 of histone H3 (H3K27), thus affecting chromatin structure and gene expression. Trimethylated H3K27 is generally associated with gene inactivation and monomethylated H3K27 with gene activation $[9,10]$.

Several studies have begun to unravel the role of JMJD3 (a member of the JmjC family) in malignancy and its clinical potential, demonstrating its involvement in a number of cancers including prostate, breast, renal, hepatocellular carcinoma, glioblastoma and melanoma [11-17]. In normal tissues, JMJD3 expression is found in the skin, tonsil, pancreas, adrenal gland and colon [18] JMJD3 promotes demethylation through its H3K27 demethylase activity, catalysing the removal of the trimethyl group from the H3K27 lysine residue $[9,19]$. The location of JMJD3 is in both the cytoplasm and nucleus, with nuclear localisation vital for effective demethylation [20]. However, the significance of cytoplasmic localization remains unclear.

JMJD3 can promote and inhibit tumorigenesis due to its dual roles on many types of cancers through binding to either promoters of oncogenes or suppressor genes enabling either a carcinogenic or a tumour suppressor role [21,22]. Most studies have investigated the nuclear expression of JMJD3 in a wide range of cancers [17,23-25]. Several investigators have also begun to recognise the demethylase independent actions of JMJD3, through mediating interactions between chromatin modifiers such as SWI/SNF chromatin-remodelling complexes and enabling gene transcription [26-28]. Additionally, Jmjd3 regulates inflammatory genes in macrophages upon microbial stimuli [19]. It appears that with JMJD3, the chromatin-remodelling function is as vital to proper gene expression as demethylase activity [26]. This again raises questions regarding the cytoplasmic expression of JMJD3. JMJD3 is reported to be an inducer of senescence by entering the cytoplasm (specifically the golgi complex) and interacting with $\mathrm{Rb}$ (retinoblastoma protein). It demethylates $\mathrm{Rb}$ (a non-histone protein), impeding its interaction with CDK4 [29]. Additionally, cytosolic JMJD3 has been reported to interact with and determine the distribution of p53 within cancer cells [30].

Previous studies on JMJD3 in mesothelioma are limited. One study evaluated mRNA expression of JMJD3 (alias KDM6B) and found high levels in pleural mesothelioma specifically in the biphasic subtype. In silico analysis however showed that JMJD3 was overexpressed only in a small cohort of mesothelioma cases [31]. We have investigated JMJD3 expression in 99 pleural mesothelioma tissues to look for its immunohistochemical expression in the three most common histological subtypes. Here, we assess for the first time the nuclear and cytoplasmic expression of JMJD3 in pleural mesothelioma, and their association with survival. 


\section{Results}

\subsection{Clinicopathological Features}

The median age of the patients at initial surgery was 75 years (range, 47-90 years). The mean duration of follow-up was 12 months (range, $2-87$ months). In total, $81 \%$ of the patients in our cohort were male and 19\% were female. The epithelioid subtype totalled 50 tumours, while 27 and 22 tumours were of the sarcomatoid and biphasic subtype, respectively. General patient characteristics for the cohort are summarised in Table 1.

Table 1. Clinicopathological parameters of 99 PM.

\begin{tabular}{cc}
\hline Clinicopathological Parameters & $\mathbf{n}(\mathbf{\%})$ \\
\hline Age at diagnosis [median years(range)] & 75 years (47-90) \\
\hline Sex & $80(81)$ \\
Male & $19(19)$ \\
Female & \\
Histological subtype & $50(51)$ \\
Epithelioid & $27(27)$ \\
Sarcomatoid & $22(22)$ \\
Biphasic & \\
Ki-67 expression & $61(67)$ \\
low & $30(33)$ \\
high & $13(13)$ \\
present & $84(87)$ \\
absent & $48(48)$ \\
\hline GATA3 IHC expression & $51(52)$ \\
low & \\
high & $93(94)$ \\
JMJD3 nuclear expression & $6(6)$ \\
low & $47(48)$ \\
high & $4(4)$ \\
NHCCL & $2(2)$ \\
\hline JMJD3 cytoplasmic expression &
\end{tabular}

\subsection{JMJD3 Expression on IHC}

Both nuclear and cytoplasmic labelling was seen in 99 pleural mesothelioma. Two pathologists scored IHC labelling, and the concordance rate of these pathologists was $82 \%$. High nuclear expression was seen in 52\%, low nuclear expression in $48 \%$, high cytoplasmic expression in 6\% and low cytoplasmic labelling in 94\% tumours (Figures 1 and 2). Within the sarcomatoid group, low nuclear expression was seen in majority of samples (89\%) and high cytoplasmic expression in only $7 \%$ (Table 2). Stromal cells showed variable expression of JMJD3, variable in most lymphocytes but present in plasma cells (Supplementary Figure S1). 


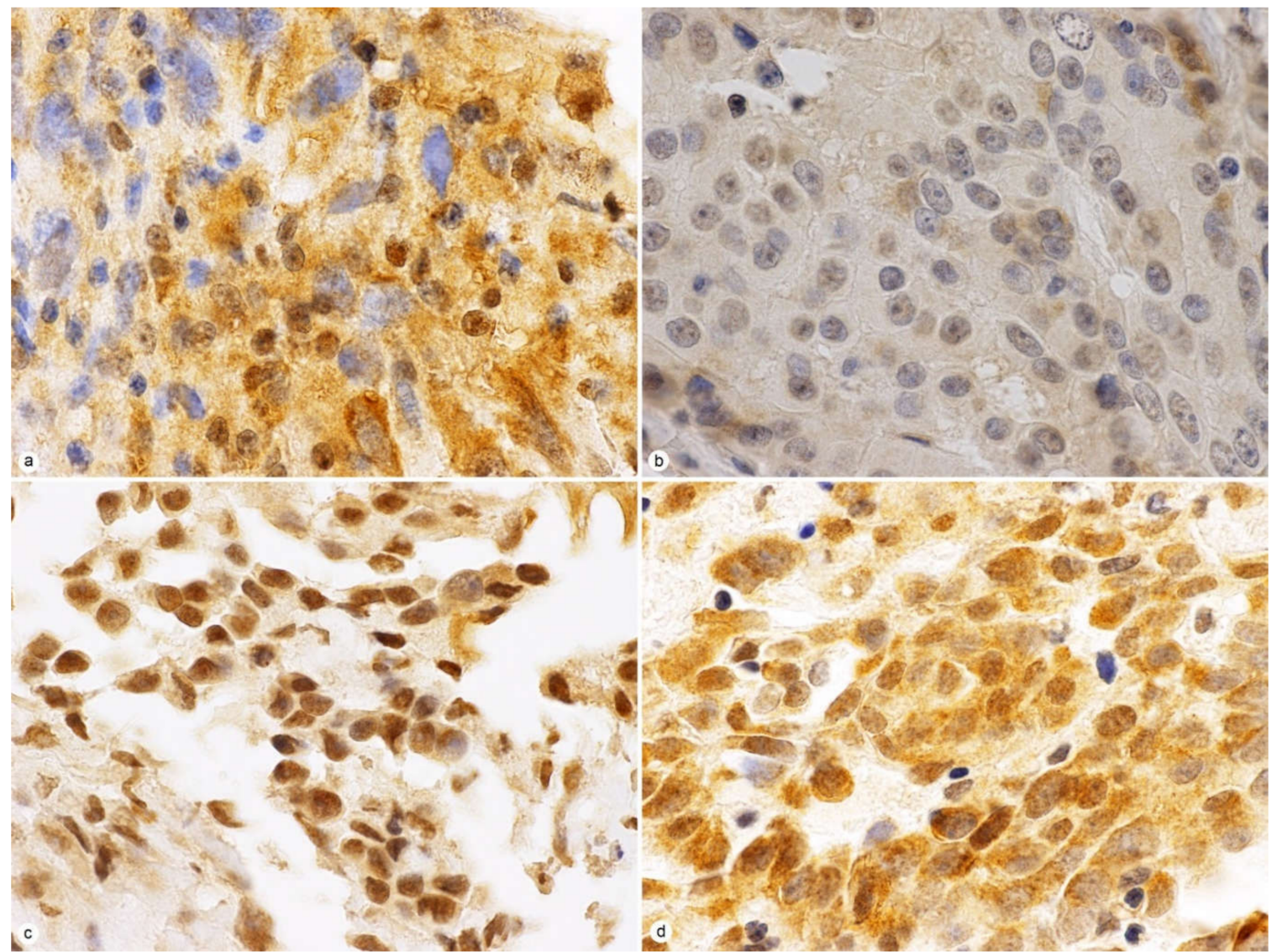

Figure 1. JMJD3 labelling by immunohistochemistry magnification 40×: (a) nuclear low and cytoplasmic high expression; (b) nuclear low and cytoplasmic low expression; (c) nuclear high and cytoplasmic low expression; (d) nuclear high and cytoplasmic high expression.

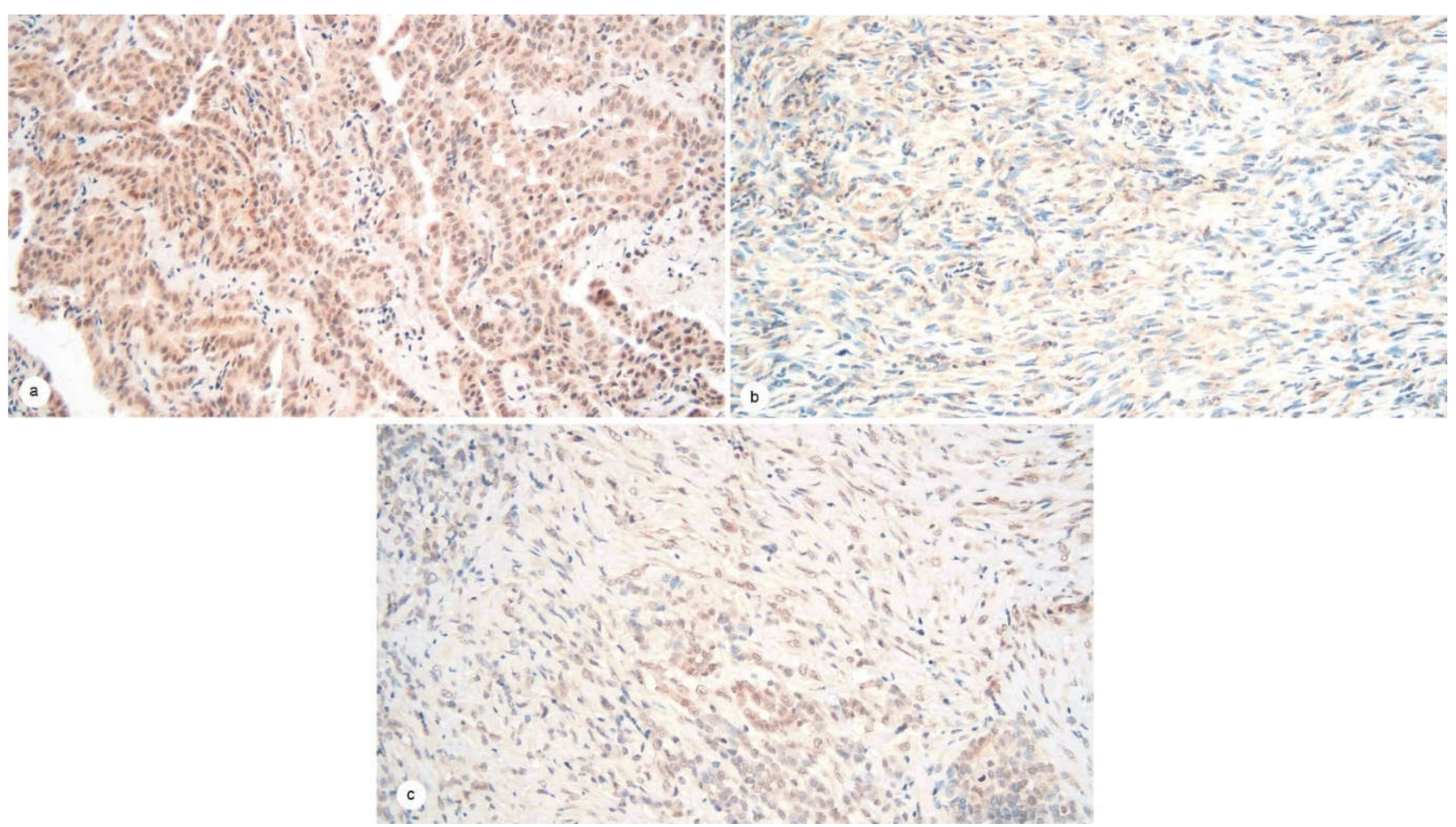

Figure 2. JMJD3 labelling by immunohistochemistry in pleural mesothelioma magnification 20×: (a) epithelioid; (b) sarcomatoid; (c) biphasic. 
Table 2. Distribution of JMJD3 in the three subtypes of mesothelioma.

\begin{tabular}{|c|c|c|c|c|c|c|c|}
\hline \multirow{2}{*}{ Subtype } & \multirow{2}{*}{$\mathbf{N}$} & \multicolumn{2}{|c|}{ Nuclear } & \multirow{2}{*}{$p$ Value } & \multicolumn{2}{|c|}{ Cytoplasmic } & \multirow{2}{*}{$p$ Value } \\
\hline & & High n (\%) & Low n (\%) & & High n (\%) & Low n (\%) & \\
\hline Total & 99 & 51 & 48 & & 6 & 93 & \\
\hline Epithelioid & 50 & $42(84)$ & $8(16)$ & $<0.001$ & $4(8)$ & $46(92)$ & 0.399 \\
\hline Sarcomatoid & 27 & $3(11)$ & $24(89)$ & & $2(7)$ & $25(93)$ & \\
\hline Biphasic & 22 & $6(27)$ & $16(73)$ & & 0 & $22(100)$ & \\
\hline
\end{tabular}

\subsection{JMJD3 Association with Survival}

When nuclear and cytoplasmic labelling were considered individually, Kaplan-Meier survival curves indicated that tumours with low nuclear expression and high cytoplasmic expression of JMJD3 had poorer survival compared to those with high nuclear and low cytoplasmic labelling ( $p=0.014$ and $p=0.041$, respectively). When nuclear and cytoplasmic expression were combined into two groups, i.e., NLCH vs. other, the NLCH group too exhibited poorer survival $(p=0.009)$. Additionally, high Ki67 expression was associated with poor prognosis $(p=0.030)$ (Figure 3$)$. No association was found between Ki67 and GATA3 or JMJD3. There was no association of GATA3 with JMJD3 IHC expression in this cohort.

\subsection{Univariate and Multivariate Analysis of JMJD3 Expression}

Although univariate analysis suggested that both nuclear and cytoplasmic JMJD3 expression had prognostic implications, multivariate analysis revealed that only high cytoplasmic expression was an independent prognostic factor. $(p=0.045$, HR: 2.463, 95\%CI (1.019-5.958)) (Table 3).

Table 3. Univariate and multivariate analysis of Mesothelioma using Cox regression.

\begin{tabular}{|c|c|c|c|c|c|c|c|c|}
\hline \multicolumn{5}{|c|}{ UVA } & \multicolumn{4}{|c|}{ MVA } \\
\hline & \multirow[t]{2}{*}{ Sig. } & \multirow[t]{2}{*}{ HR } & \multicolumn{2}{|c|}{$95.0 \%$ CI for $\operatorname{Exp}(B)$} & \multirow[t]{2}{*}{ Sig. } & \multirow[t]{2}{*}{ HR } & \multicolumn{2}{|c|}{$95.0 \%$ CI for $\operatorname{Exp}(B)$} \\
\hline & & & Lower & Upper & & & Lower & Upper \\
\hline Ki67 & 0.036 & 1.631 & 1.033 & 2.576 & 0.009 & 1.959 & 1.187 & 3.233 \\
\hline Nuclear JMJ & 0.018 & 1.622 & 1.088 & 2.42 & 0.369 & 1.389 & 0.678 & 2.844 \\
\hline Cyto JMJ & 0.052 & 2.316 & 0.992 & 5.405 & 0.045 & 2.463 & 1.019 & 5.958 \\
\hline Gata3 & 0.55 & 1.207 & 0.651 & 2.237 & 0.563 & 0.811 & 0.398 & 1.651 \\
\hline \multicolumn{9}{|l|}{ Subtype: } \\
\hline Ref Epithelioid & 1 & & & & 1 & & & \\
\hline Sarcomatoid & 0.002 & 2.157 & 1.338 & 3.478 & 0.083 & 2.014 & 0.914 & 4.437 \\
\hline Biphasic & 0.311 & 1.305 & 0.78 & 2.183 & 0.399 & 1.404 & 0.638 & 3.088 \\
\hline
\end{tabular}

HR, Hazard ratio; CI, Confidence interval. Included are 99 patients. 


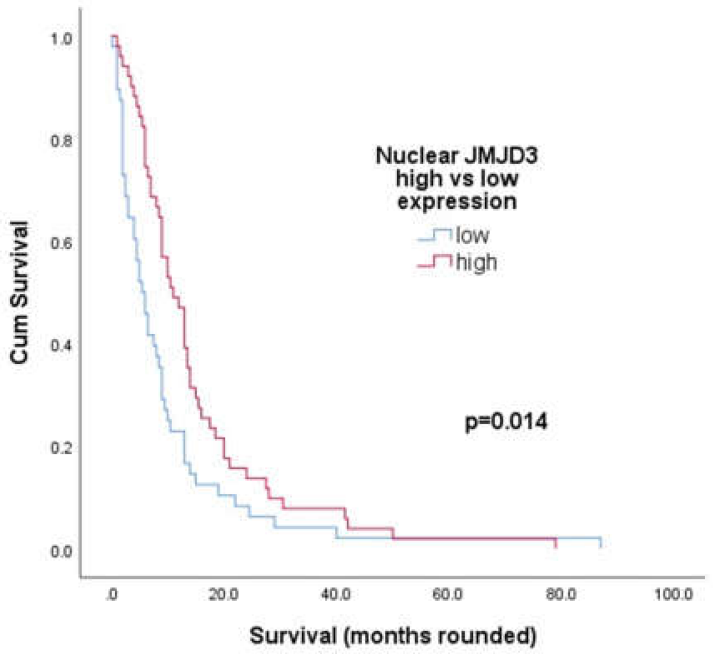

(a)

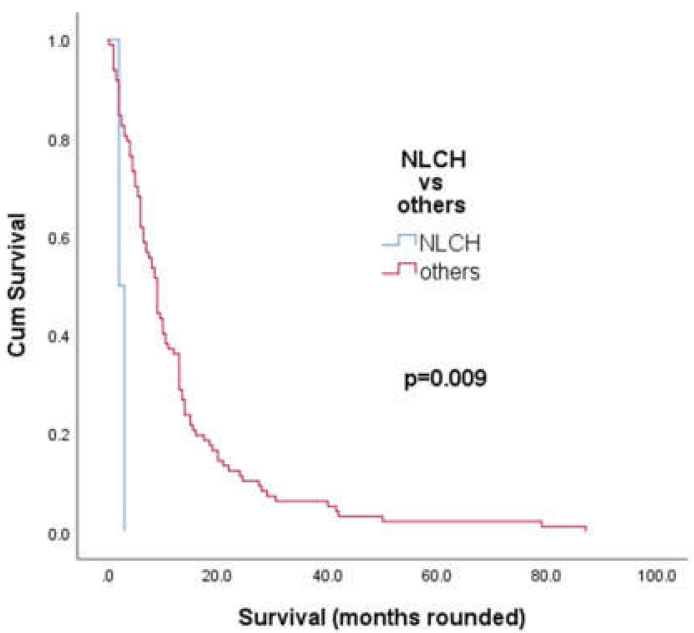

(c)

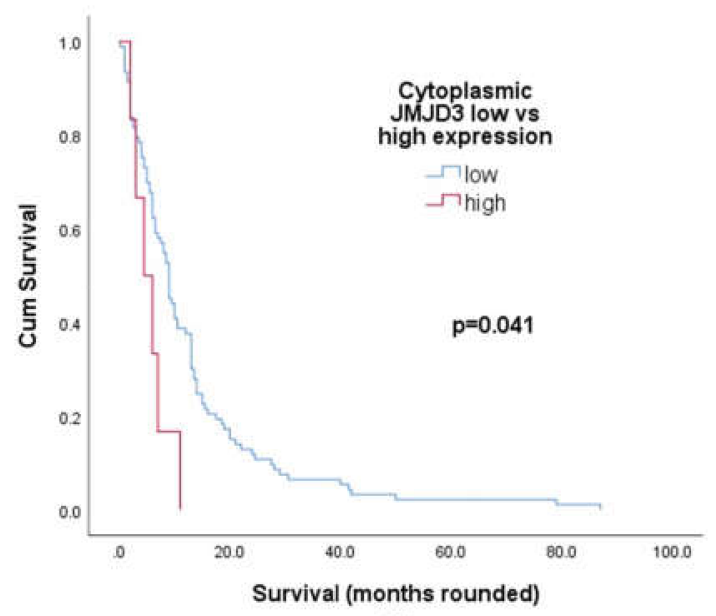

(b)

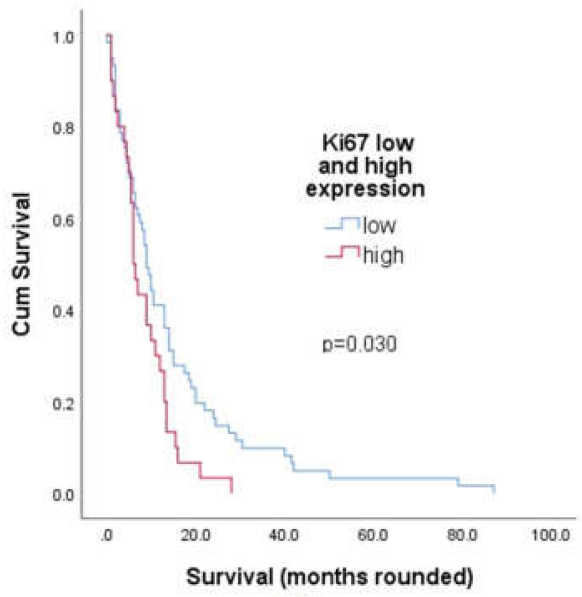

(d)

Figure 3. Kaplan-Meier survival curve of JMJD3 and Ki67 IHC expression in this cohort of 99 pleural mesothelioma patients. (a) The median overall survival was 5.5 months (95\% CI 3.56-7.44) for patients with low nuclear expression, ( $n=48)$, versus a median overall survival of 11 months (95\% CI 7.89-14.11) for patients with high nuclear expression $(n=51)$; (b) Median overall survival of 9 months (95\% CI 7.43-10.56) for patients with low cytoplasmic expression, $(n=93)$, versus a median overall survival of 4.5 months (95\% CI 0.89-8.10) for patients with high cytoplasmic expression $(n=6)$; (c) Median overall survival of 2.5 months (95\% CI 2.0-3) for patients with NLCH expression, $(n=2)$, versus a median overall survival of 9 months (95\% CI 7.69-10.30) for all other groups, $(n=97)$; (d) Median overall survival of 9 months (95\% CI 7.1-10.9) for patients with low Ki67expression, $(n=61)$, versus a median overall survival of 6 months (95\% CI 4.6-7.3) for patients with high nuclear expression, $(n=30)$.

\section{Discussion}

Several studies have analysed the expression of JMJD3 in a wide range of cancers. Shuman $\mathrm{Li}$ et al. found that sarcomatoid lung cancers with positive nuclear expression of JMJD3 had lower survival and hence poorer prognosis [23]. Contradictory findings in pancreatic cancer suggest that loss of JMJD3 is associated with aggressiveness [32]. Therefore, the prognostic significance of JMJD3 across various tumours is not uniform as there is discordance in the results JMJD3 protein expression among different tumours. In the majority of these studies only nuclear expression was considered [17,23-25].

We therefore decided to investigate the significance of both nuclear and cytoplasmic JMJD3 expression on immunohistochemistry in pleural mesothelioma. We found that nearly $48 \%$ of tumours in our cohort had low nuclear labelling while only $6 \%$ had high 
cytoplasmic labelling with JMJD3. Our study revealed that loss of nuclear labelling of JMJD3 is seen in a large proportion of sarcomatoid pleural mesothelioma. $(p<0.001 \mathrm{Chi}$ Square analysis). Kaplan-Meier survival curves indicated that tumours in our cohort with low nuclear expression were associated with poor prognosis compared to tumours with high nuclear expression $(p<0.014)$. Additionally, higher cytoplasmic expression was found in only $6 \%$ tumours and correlated with poor prognosis in our cohort $(p<0.041)$. Furthermore, tumours were grouped based on combinations of IHC labelling as NLCH versus others which revealed that the NLCH group had significantly poor prognosis compared to others $(p=0.009)$. Finally, we investigated both nuclear and cytoplasmic labelling of JMJD3 for any prognostic significance.

On multivariate analysis, however, only high cytoplasmic labelling independently portended poor prognosis ( $p=0.038, \mathrm{HR}=2.581,95 \% \mathrm{CI}$ : $1.054-6.321)$. JMJD3 is a histone demethylase specific for $\mathrm{H} 3 \mathrm{~K} 27 \mathrm{me} 3 / \mathrm{me} 2$ that switches a gene from its repressive state to an active form. Independent of its methylase activity, JMJD3 also has a role in chromatin remodelling and in cellular senescence. JMJD3 can act as both a tumour suppressor and an oncogene depending on the cellular context. JMJD3 is present both in the nucleus and cytoplasm, although nuclear localization is required for effective demethylase activity affecting the methylation status of H3K27 and impacting on gene expression and cellular function [20]. Senescent cells maintain some genes in a suppressed or repressed state that are essential for cell cycle progression. Cellular senescence involves the formation of SASP (senescence-associated secretory phenotype) characterised by secretion of inflammatory cytokines, growth factors, and proteases [10]. In mesothelioma, the SASP is considered capable of tumour initiation and responsible for the formation of chemoresistant tumour cells [33]. The function of JMJD3 lies at the intersection of senescence and cancer progression. During the process of senescence, JMJD3 is transported to the cytoplasm and demethylates RB an important regulator of the senescence pathway [29]. We hypothesize that high cytoplasmic expression of JMJD3 relates to mesothelioma cells with high SASP activity promoting survival of tumour cells.

One major limitation of our study was the small number of cases $(n=6)$ that showed high cytoplasmic labelling with JMJD3. Mesothelioma is a rare cancer but the fact that in this pilot study high cytoplasmic expression remained statistically significant may indicate a large effect. We do not have a validation cohort currently. However, analysis of JMJD3 expression in a larger cohort of mesothelioma patients, and correlation with therapy, would aid in understanding the significance and extent of demethylase-independent activity of JMJD3 within the cytoplasm in pleural mesothelioma.

\section{Materials and Methods}

\subsection{Patient Population}

This study utilised a cohort of 99 patient archival tissues diagnosed at the Department of Anatomical Pathology at Flinders Medical Centre between the years of 1991 and 2013. Patient inclusion criteria consisted of a histological diagnosis of mesothelioma, sufficient tissue for analysis and access to clinical follow-up information. This work was approved by The Southern Adelaide Clinical Human Research Ethics Committee (approval number HREC/19/SAC/26).

\subsection{Tissue Microarrays and JMJD3 Immunohistochemical Analysis}

Diagnosis was established by light microscopy and positive IHC labelling for epithelioid mesothelial markers according to current guidelines (at least two antibodies for mesothelial markers CK5/6, calretinin, WT1, D2-40, thrombomodulin and HBME-1) and at least two carcinoma markers (CEA, TTF-1, MOC31, BerEp4, and Claudin4) by two board-certified pathologists with interests in lung pathology [3-5]. Antibodies were selected to exclude an epithelioid hemangioendothelioma or an epithelioid angiosarcoma supplemented by radiological demonstration that the tumour was pleural with no imaging evidence of extra serosal tumour [34-36]. BAP1 was assessed retrospectively in this cohort 
and hence has been published [37]. Tissue microarrays consisting of 99 pleural mesothelioma tissues were constructed. Four cores of $1 \mathrm{~mm}$ diameter were arrayed to represent each tumour in order to reach concordance with full sections [38]. The mesothelioma microarrays were initially stained with hematoxylin and eosin and histopathologic evaluation was performed. Next, paraffin sections were cut, deparaffinised and rehydrated using graded concentration of xylene and ethanol. Slides were immersed in $1 \% \mathrm{H}_{2} \mathrm{O}_{2}$ and $50 \%$ ethanol to quench endogenous peroxidases. Heat-induced alkaline retrieval was performed using Dako Target Retrieval Solution. Sections were blocked with $10 \%$ normal goat serum in TBS for 30 min. Sections were coated with 1:1000 rabbit polyclonal JMJD3 antibody (Invitrogen) Catalog number PA5-32192 and incubated at $4{ }^{\circ} \mathrm{C}$ overnight. The Novolink Max Polymer Detection kit (Leica Biosystems, Nussloch, Germany) and DAB+ Chromogen System (Dako Australia Pty. Ltd., Sydney, NSW, Australia) were used for detection, before haematoxylin counterstaining. IHC expression was evaluated using an Olympus BX46 microscope (Olympus, Tokyo, Japan) with a standard eyepiece of $22 \mathrm{~mm}$ diameter. Normal colon tissue was used as a positive control and incubation without the primary antibody was used as a negative control. Diagnostic clinical procedures relating to diagnosis of the cases were performed in a NATA-approved laboratory using QAP validated tests.

\subsection{JMJD3 Scoring}

Nuclear and cytoplasmic expression of JMJD3 were first assessed separately. Staining intensity for both nuclear and cytoplasmic components was scored as follows: no or low staining recognisable as a blush at $10 \times$ and $20 \times(0)$; moderate staining visible as a blush at $4 \times$ magnification (1) or intense staining visible as prominent staining at $4 \times(2)$. Percentage of cells stained positively was scored between 0-100. A final H score (range 0-300) was obtained by adding the sum of scores obtained for each intensity and proportion of area stained $(\mathrm{H}$ score $=\mathrm{I} 1 \times \mathrm{P} 1+\mathrm{I} 2 \times \mathrm{P} 2+\mathrm{I} 3 \times \mathrm{P} 3$, etc.). The Xtile software (Yale University, New Haven, CT, USA) was used to generate cutoffs based on outcome to stratify tumours into high and low expression groups.

Based on the cutoffs, mesothelioma cases were sub-grouped into two groups, one with low or complete absence of nuclear staining $(\mathrm{H}$ score $<10)$; the other group $(\mathrm{H}$ score $>10)$ was defined as high expression for nuclear JMJD3. Cytoplasmic expression was also similarly obtained using $X$ tile cutoffs of 180 into two groups: one with low or complete absence of cytoplasmic staining ( $\mathrm{H}$ score $<180)$; the other group $\mathrm{H}$ score $>180$ was defined as high expression for cytoplasmic JMJD3. The mean and standard deviation [Mean(SD)] of histoscores for nuclear expression of JMJD3 in our cohort was 34.27(47.9) and for the cytoplasmic expression of JMJD3 was 83.17(54.8). Histoscores for JMJD3 expression ranged from 0-180 for nuclear expression and 0-260 for cytoplasmic expression. Next, tumours were stratified into two groups: Nuclear low and cytoplasmic high (NLCH) and others (including nuclear low cytoplasmic low- NLCL, nuclear high cytoplasmic high-NHCH and nuclear high cytoplasmic low-NHCL). The Xtile software was also used to obtain cutoff values for Ki67 expression in our cohort.

\subsection{Statistical Analysis}

Chi Square analysis was performed to investigate factors associated with JMJD3 expression. Kaplan-Meier curves were used to analyse survival, and Cox Regression univariate and multivariant analysis were used to assess prognostic significance. SPSS v. 23 software was used for all statistical analysis.

Supplementary Materials: The following is available online at https:/ /www.mdpi.com/article/10.339 0/jmp2030019/s1. Figure S1: JMJD3 IHC expression in stromal cells of pleural mesothelioma.

Author Contributions: Conceptualization, S.K. and S.P.; ethics, S.P., S.K. and L.R.-N.; methodology, S.K., S.P., A.J.H. and L.R.-N.; data curation, L.R.-N., A.J.H. and M.H.; statistics, S.P.; formal analysis, S.P. and L.R.-N.; writing-original draft preparation, S.P. and L.R.-N.; writing-review and editing, S.P., S.K. and L.R.-N. All authors have read and agreed to the published version of the manuscript. 
Funding: This research was funded by the Douglas Henderson AO bequest fund of Flinders University.

Institutional Review Board Statement: This work was approved by The Southern Adelaide Clinical Human Research Ethics Committee (approval number HREC/19/SAC/26).

Informed Consent Statement: Not applicable.

Data Availability Statement: Not applicable.

Conflicts of Interest: Sonja Klebe prepares medicolegal reports for the courts of Australia on the diagnosis and causation of occupational lung disease, outside of the submitted work. The authors report no other conflict of interest in this work.

\section{References}

1. World Health Organization. Mesothelioma Fact Sheet. Available online: Gco.iarc.fr/today/data/factsheets/cancers/18 -Mesothelioma-fact-sheet.pdf (accessed on 18 June 2021).

2. Australian Institute of Health and Welfare. Mesothelioma in Australia 2019; AIHW: Canberra, Australia, 2020.

3. Meyerhoff, R.R.; Yang, C.F.; Speicher, P.J.; Gulack, B.C.; Hartwig, M.G.; D'Amico, T.A.; Harpole, D.H.; Berry, M.F. Impact of mesothelioma histologic subtype on outcomes in the Surveillance, Epidemiology, and End Results database. J. Surg. Res. 2015, 196, 23-32. [CrossRef] [PubMed]

4. Chapel, D.B.; Schulte, J.J.; Husain, A.N.; Krausz, T. Application of immunohistochemistry in diagnosis and management of malignant mesothelioma. Transl. Lung Cancer Res. 2020, 9, S3-S27. [CrossRef] [PubMed]

5. Galateau-Salle, F.; Churg, A.; Roggli, V.; Travis, W.D.; World Health Organization Committee for Tumors of the Pleura. The 2015 World Health Organization Classification of Tumors of the Pleura: Advances since the 2004 Classification. J. Thorac. Oncol. 2016, 11, 142-154. [CrossRef] [PubMed]

6. Husain, A.N.; Colby, T.V.; Ordonez, N.G.; Allen, T.C.; Attanoos, R.L.; Beasley, M.B.; Butnor, K.J.; Chirieac, L.R.; Churg, A.M.; Dacic, S.; et al. Guidelines for Pathologic Diagnosis of Malignant Mesothelioma 2017 Update of the Consensus Statement From the International Mesothelioma Interest Group. Arch. Pathol. Lab. Med. 2018, 142, 89-108. [CrossRef]

7. Marchevsky, A.M.; LeStang, N.; Hiroshima, K.; Pelosi, G.; Attanoos, R.; Churg, A.; Chirieac, L.; Dacic, S.; Husain, A.; Khoor, A.; et al. The differential diagnosis between pleural sarcomatoid mesothelioma and spindle cell/pleomorphic (sarcomatoid) carcinomas of the lung: Evidence-based guidelines from the International Mesothelioma Panel and the MESOPATH National Reference Center. Hum. Pathol. 2017, 67, 160-168. [CrossRef]

8. Eccher, A.; Girolami, I.; Lucenteforte, E.; Troncone, G.; Scarpa, A.; Pantanowitz, L. Diagnostic mesothelioma biomarkers in effusion cytology. Cancer Cytopathol. 2021. [CrossRef]

9. Agger, K.; Cloos, P.A.; Christensen, J.; Pasini, D.; Rose, S.; Rappsilber, J.; Issaeva, I.; Canaani, E.; Salcini, A.E.; Helin, K. UTX and JMJD3 are histone H3K27 demethylases involved in HOX gene regulation and development. Nature 2007, 449, 731-734. [CrossRef]

10. Perrigue, P.M.; Najbauer, J.; Barciszewski, J. Histone demethylase JMJD3 at the intersection of cellular senescence and cancer. Biochim. Biophys. Acta 2016, 1865, 237-244. [CrossRef]

11. Hong, Z.; Li, H.; Li, L.; Wang, W.; Xu, T. Different expression patterns of histone H3K27 demethylases in renal cell carcinoma and bladder cancer. Cancer Biomark 2017, 18, 125-131. [CrossRef]

12. Ma, J.; Wang, N.; Zhang, Y.; Wang, C.; Ge, T.; Jin, H.; Deng, X.; Huo, X.; Gu, D.; Ge, Z.; et al. KDM6B Elicits Cell Apoptosis by Promoting Nuclear Translocation of FOXO1 in Non-Small Cell Lung Cancer. Cell Physiol. Biochem. 2015, 37, 201-213. [CrossRef]

13. Ramadoss, S.; Chen, X.; Wang, C.Y. Histone demethylase KDM6B promotes epithelial-mesenchymal transition. J. Biol. Chem. 2012, 287, 44508-44517. [CrossRef]

14. Tokunaga, R.; Sakamoto, Y.; Nakagawa, S.; Miyake, K.; Izumi, D.; Kosumi, K.; Taki, K.; Higashi, T.; Imamura, Y.; Ishimoto, T.; et al. The Prognostic Significance of Histone Lysine Demethylase JMJD3/KDM6B in Colorectal Cancer. Ann. Surg. Oncol. 2016, 23, 678-685. [CrossRef]

15. Zhang, Y.; Shen, L.; Stupack, D.G.; Bai, N.; Xun, J.; Ren, G.; Han, J.; Li, L.; Luo, Y.; Xiang, R.; et al. JMJD3 promotes survival of diffuse large B-cell lymphoma subtypes via distinct mechanisms. Oncotarget 2016, 7, 29387-29399. [CrossRef]

16. Zou, S.; Zhang, D.; Xu, Z.; Wen, X.; Zhang, Y. JMJD3 promotes the epithelial-mesenchymal transition and migration of glioma cells via the CXCL12/CXCR4 axis. Oncol. Lett. 2019, 18, 5930-5940. [CrossRef]

17. Tang, B.; Qi, G.; Tang, F.; Yuan, S.; Wang, Z.; Liang, X.; Li, B.; Yu, S.; Liu, J.; Huang, Q.; et al. Aberrant JMJD3 Expression Upregulates Slug to Promote Migration, Invasion, and Stem Cell-Like Behaviors in Hepatocellular Carcinoma. Cancer Res. 2016, 76, 6520-6532. [CrossRef]

18. The Human Protein Atlas. Available online: https://www.proteinatlas.org/ENSG00000132510-KDM6B/tissue (accessed on 11 March 2021).

19. De Santa, F.; Narang, V.; Yap, Z.H.; Tusi, B.K.; Burgold, T.; Austenaa, L.; Bucci, G.; Caganova, M.; Notarbartolo, S.; Casola, S.; et al. Jmjd3 contributes to the control of gene expression in LPS-activated macrophages. EMBO J. 2009, 28, 3341-3352. [CrossRef]

20. Kamikawa, Y.F.; Donohoe, M.E. The localization of histone H3K27me3 demethylase Jmjd3 is dynamically regulated. Epigenetics 2014, 9, 834-841. [CrossRef] 
21. Agger, K.; Cloos, P.A.; Rudkjaer, L.; Williams, K.; Andersen, G.; Christensen, J.; Helin, K. The H3K27me3 demethylase JMJD3 contributes to the activation of the INK4A-ARF locus in response to oncogene- and stress-induced senescence. Genes Dev. 2009, 23, 1171-1176. [CrossRef]

22. Chen, X.; Xiao, X.; Guo, F. Roles of H3K27me3 Demethylase JMJD3 in Inflammation and Cancers. J. Rare Dis. Res. Treat. 2019, 4, 71-76. [CrossRef]

23. Li, S.; Jiang, L.; He, Q.; Wei, W.; Wang, Y.; Zhang, X.; Liu, J.; Chen, K.; Chen, J.; Xie, D. The Prognostic Significance of JMJD3 in Primary Sarcomatoid Carcinoma of The Lung, a Rare Subtype of Lung Cancer. OncoTargets Ther. 2019, 12, 9385-9393. [CrossRef]

24. Li, S.H.; Lu, H.I.; Chen, Y.H.; Lo, C.M.; Huang, W.T.; Tien, W.Y.; Lan, Y.C.; Tsai, H.T.; Chen, C.H. JMJD3 expression is an independent prognosticator in patients with esophageal squamous cell carcinoma. Surgery 2019, 165, 946-952. [CrossRef]

25. Xu, Z.; Xia, Y.; Xiao, Z.; Jia, Y.; Li, L.; Jin, Y.; Zhao, Q.; Wan, L.; Yi, T.; Yu, Y.; et al. Comprehensive profiling of JMJD3 in gastric cancer and its influence on patient survival. Sci. Rep. 2019, 9, 868. [CrossRef] [PubMed]

26. Miller, S.A.; Mohn, S.E.; Weinmann, A.S. Jmjd3 and UTX play a demethylase-independent role in chromatin remodeling to regulate T-box family member-dependent gene expression. Mol. Cell 2010, 40, 594-605. [CrossRef]

27. Ohguchi, H.; Harada, T.; Sagawa, M.; Kikuchi, S.; Tai, Y.T.; Richardson, P.G.; Hideshima, T.; Anderson, K.C. KDM6B modulates MAPK pathway mediating multiple myeloma cell growth and survival. Leukemia 2017, 31, 2661-2669. [CrossRef]

28. Salminen, A.; Kaarniranta, K.; Hiltunen, M.; Kauppinen, A. Histone demethylase Jumonji D3 (JMJD3/KDM6B) at the nexus of epigenetic regulation of inflammation and the aging process. J. Mol. Med. 2014, 92, 1035-1043. [CrossRef]

29. Zhao, L.; Zhang, Y.; Gao, Y.; Geng, P.; Lu, Y.; Liu, X.; Yao, R.; Hou, P.; Liu, D.; Lu, J.; et al. JMJD3 promotes SAHF formation in senescent WI38 cells by triggering an interplay between demethylation and phosphorylation of RB protein. Cell Death Differ. 2015, 22, 1630-1640. [CrossRef]

30. Sola, S.; Xavier, J.M.; Santos, D.M.; Aranha, M.M.; Morgado, A.L.; Jepsen, K.; Rodrigues, C.M. p53 interaction with JMJD3 results in its nuclear distribution during mouse neural stem cell differentiation. PLoS ONE 2011, 6, e18421. [CrossRef]

31. Cregan, S.; Breslin, M.; Roche, G.; Wennstedt, S.; MacDonagh, L.; Albadri, C.; Gao, Y.; O’Byrne, K.J.; Cuffe, S.; Finn, S.P.; et al. Kdm6a and Kdm6b: Altered expression in malignant pleural mesothelioma. Int. J. Oncol. 2017, 50, 1044-1052. [CrossRef]

32. Yamamoto, K.; Tateishi, K.; Kudo, Y.; Sato, T.; Yamamoto, S.; Miyabayashi, K.; Matsusaka, K.; Asaoka, Y.; Ijichi, H.; Hirata, Y.; et al. Loss of histone demethylase KDM6B enhances aggressiveness of pancreatic cancer through downregulation of C/EBPalpha. Carcinogenesis 2014, 35, 2404-2414. [CrossRef]

33. Canino, C.; Mori, F.; Cambria, A.; Diamantini, A.; Germoni, S.; Alessandrini, G.; Borsellino, G.; Galati, R.; Battistini, L.; Blandino, R.; et al. SASP mediates chemoresistance and tumor-initiating-activity of mesothelioma cells. Oncogene 2012, 31, 3148-3163. [CrossRef]

34. Lin, B.T.-Y.; Colby, T.; Gown, A.M.; Hammar, S.P.; Mertens, R.B.; Churg, A.; Battifora, H. Malignant vascular tumors of the serous membranes mimicking mesothelioma: A report of 14 cases. Am. J. Surg. Pathol. 1996, 20, 1431-1439. [CrossRef] [PubMed]

35. Kao, Y.-C.; Chow, J.-M.; Wang, K.-M.; Fang, C.-L.; Chu, J.-S.; Chen, C.-L. Primary pleural angiosarcoma as a mimicker of mesothelioma: A case report. Diagn. Pathol. 2011, 6, 1-5. [CrossRef]

36. Del Frate, C.; Mortele, K.; Zanardi, R.; Hunsaker, A.R.; Nikpoor, N.; Cibas, E.S.; Silverman, S.G. Pseudomesotheliomatous angiosarcoma of the chest wall and pleura. J. Thorac. Imaging 2003, 18, 200-203. [CrossRef] [PubMed]

37. Pulford, E.; Huilgol, K.; Moffat, D.; Henderson, D.W.; Klebe, S. Malignant Mesothelioma, BAP1 Immunohistochemistry, and VEGFA: Does BAP1 Have Potential for Early Diagnosis and Assessment of Prognosis? Dis. Markers 2017, 2017, 1310478. [CrossRef]

38. Kao, S.C.; Lee, K.; Armstrong, N.J.; Clarke, S.; Vardy, J.; van Zandwijk, N.; Reid, G.; Burn, J.; McCaughan, B.C.; Henderson, D.W.; et al. Validation of tissue microarray technology in malignant pleural mesothelioma. Pathology 2011, 43, 128-132. [CrossRef] 